\title{
Original $\mid$ THE EFFICACY AND TOXICITY OF OXALIPLATIN, 5-FLUOROURACIL Article AND LEUCOVORIN AS ADJUVANT TREATMENT IN PATIENTS WITH COLON CANCER
}

\author{
Hanan seleem, Tamer El-Nahas, Magda Mostafa \\ Department of Clinical oncology
}

\begin{abstract}
Background: Although surgical resection is still the only curative maneuver in the treatment of colon cancer, yet efforts have proved that systemic chemotherapy in the adjuvant setting improves the curative rate for those patients with localized colon cancer. The combination of 5-fluorouracil (5-Fu) and leucovorin (LV) was the reference treatment. However the advantage of infusional 5-Fu/LV with oxaliplatin (FolFox-4) as adjuvant treatment for high risk colon cancer patients may change the paradigm soon. In this study we presented our treatment results for patients with colon cancer treated with FolFox- 4 regimen as adjuvant treatment at Kasr Al Aini Hospital.

Patients and Methods: We enrolled 55 patients who had undergone curative resection for sage II and III colon cancer to receive FolFox-4 regimen for six months. The primary end point was disease -free survival

Results: Fifty five patients were included in this study. At the time of analysis (median follow up 18 months) 9 patients (16\%) had disease relapse or metastasis. At 2.5 years DFS rates and OAS rates were $75 \%$ and $80 \%$, respectively. The treatment was generally well tolerated with most common grade 1 treatment-related adverse event being peripheral neuropathy and hematological toxicity.

Conclusion: It is now acceptable to consider combination chemotherapy with FolFox -4 as adjuvant treatment for high risk colon cancer patients after curative surgery at Kasr El Ainy center of Radiation Oncology and nuclear medicine.
\end{abstract}

Key Words: Colon cancer, adjuvant treatment, FolFox

Corresponding Author: Hanan seleem

E-mail: elnahas, @sphinx cure.com

\section{INTRODUCTION}

Colorectal cancer (CRC) accounts for $10 \%$ to $15 \%$ of all cancer and is the second leading cause of cancer death in western countries. Approximately half of all patients develop metastatic disease ${ }^{1}$.

The 5-years survival for all patients has improved significantly from $50 \%$ in $1974-1976$ to $62 \%$ in $1992-$ $1998^{2}$.

Surgery is the primary curative modality in patients with localized (CRC), however, the risk of recurrences is still high in many patients. Postoperative adjuvant chemotherapy for patients with high risk colon cancer has been demonstrated to improve patient outcome and become the standard of care ${ }^{3-10}$.

A clinical trial (Multicenter international study of oxaliplatin, 5-fluorouraicl (5-FU), leucovorin (LV) as adjuvant treatment of colon cancer
(MOSAIC) trial reported ${ }^{8}$ revealed that adding oxaliplatin to a regimen of bolus and continuous infusion 5-fluorouracil (5-Fu) combined with LV (FolFox-4) produced a significant improvement in 3-year disease -free survival (DFS) compared with the same regimen of $5-\mathrm{Fu} / \mathrm{LV}$ administered alone ${ }^{8}$. Continued follow-up has demonstrated that the advantage for FloFox 4 has been maintained ${ }^{9}$. At a median follow-up of 6 years, the recently updated MOSAIC trial demonstrates a significant benefit of FOLFOX in OS for stage III patients (73\% vs. $68 \%, p=0.029)$.DFS rates with FOLFOX versus 5 -FU/LV, at 5 years follow-up, are consistent (73\% vs.67.4\%, HR: 0.80, $\mathrm{P}=0.003$ ). However, there is no OS benefit indicated for the stage II colon cancer patients ${ }^{11}$.

AT 2005 the same results have been achieved in (NSABP) trial ${ }^{10}$. 
The aim of our study is to evaluate the efficacy and toxicity of treatment with FolFox-4 regimen in the postoperative setting $\mathrm{y}$. The primary end point is disease- free survival.

\section{PATIENTS AND METHODS}

\section{PATIENT Eligibility:}

1. Patients eligible for this trial had either high risk stage II (T3, 4, No, Mo) or stage III (T1-4, N1-2, MO) colon cancer and had complete surgical resection with no evidence of residual malignant disease within 42 days.

2. Patients age range (18-75 years) with performance status score up to 2 (ECOG).

3. Adequate hematological, renal and hepatic functions were required

4. Patients with clinically significant peripheral neuropathy (grade 2or higher) according to National Cancer Institute Common Toxicity Criteria version $2.0^{12}$, patients with history of colon cancer or other invasive cancer were excluded.

5. No prior chemotherapy or radiotherapy.

6. Patient consent was obtained for all study group.

\section{Pretreatment evaluation and follow up:}

1. All patients underwent a history and physical examination, neurological assessment, complete blood cell count. (CBC) chemistry tests for liver and kidney functions, abdominal imaging (computed Tomography scan (CT) or magnetic resonance imaging scan (MRI)). Carcinoembryonic antigen test (CEA) and endoscopy before therapy.

2. Follow-up consisted of periodic history and physical examination, neurological assessment, CBC, liver and kidney function tests and CEA before firsts cycle during treatment and then every two months for two years and every 6 months for another 3 years. Abdominal imaging (CT or MRI) is done every 6 months, or if indicated by patient symptoms, or laboratory abnormalities. Neurological adverse effects were $\mathrm{o}$ be reported at each visit during follow -up and were assessed with the use of the neurosensory section of the Common Toxicity Criteria of the National Cancer Institute, version 1.
3. Patients had colonoscopy after 1 year and thereafter as indicated.

\section{Treatment:}

Oxaliplatin $85 \mathrm{mg} / \mathrm{m} 2$ was administered as intravenous infusion (IV) over 2 hours, at the same time with leucovorin (LV) in $200 \mathrm{mg} / \mathrm{m} 2$ as IV over 2hrs on day 1 then 5 -fluorouracil (5-Fu) was given first as a bolus at dose of $400 \mathrm{mg} / \mathrm{m} 2$ and then as infusion of $600 \mathrm{mg} / \mathrm{m} 2$ over 22hrs. On day $2 \mathrm{LV}$ and 5-Fu bolus and 5-Fu infusion over 22 hrs were repeated with the same dose.

The cycle was repeated every 2 weeks for total of 12 cycles. The above regimen was given as in-patient, preceded by $8 \mathrm{mg}$ ondansetron IV as anti-emetic drug. Adverse effects were graded according to the Common Toxicity Criteria of the National Cancer Institute (NCI), version 1. According to these criteria, a score of 1 indicates mild adverse effects, a score of 2 moderate adverse effects, a score of 3 severe adverse effects and a score of 4 life- threatening adverse effects.

The dose of oxaliplatin was reduced to $75 \mathrm{mg} /$ $\mathrm{m} 2$ in the event of persistent paresthesias (at least 14 days), temporary painful paresthesias or functional impairment. Together with reductions in the dose of oxaliplatin, the bolus dose of fluorouracil was reduced to $300 \mathrm{mg} / \mathrm{m} 2$ and infusion to $500 \mathrm{mg} / \mathrm{m} 2$ in event of grade 3 or 4 neutropenia and or thrombocytopenia and, diarrhea or stomatitis.

\section{Statistical analysis:}

Data were statistically described in terms of mean-standard $( \pm S D)$, frequencies (number of cases) and relative frequencies (percentages) when appropriate. Uni-variate and multivariate analysis models were used to test for the preferential effect of the independent variable(s) on overall survival time and disease free survival time. The primary efficacy variable was disease-free survival (DFS), defined as the time from treatment to relapse or death, whichever occurred first. Secondary end points were safety, including adverse effects and overall survival (OAS), measured from the time of treatment to death from any cause. Survival analysis was done for the different outcome measures using Kaplan Maier statistics with the corresponding graphs. 
A probability value (p-value) less than 0.05 was considered statistically significant. All statistical calculations were done using computer programs Microsoft Excel version7 (Microsoft Corporation, NY, USA) and SPSS (Statistical package for the Social Science, SPSS Inc., Chicago, IL, USA) version13 for Microsoft Windows.

\section{RESULTS}

Between September 2005 and December 2007, 55 patients were enrolled to receive FU/LV plus oxaliplatin at KASR AL AINI Oncology Center. The patient's characteristics are shown in Table (1).

The median age was 35 years (range from 18 to 73 years), most of patients were under 40 years (56.4\%).

Twenty seven percent of patients presented with bowel obstruction. Right side tumors and pathological grade 2 were the most common findings (54.6\% and $72.7 \%)$, respectively. Two patients (3.6\%) presented with signet ring pathological subtype. Thirty patients (54.5\%) had stage III disease. The median number of cycles of chemotherapy was 8 cycles (range: 6-12 cycles), $83.6 \%$ of the patients received the planned 12 cycles.

The median dosage of oxaliplatin was $72 \mathrm{mg} /$ week. The planned dose of oxaliplatin and 5-Fu were received in $85.5 \%$ of patients, while $14.5 \%$ patients who experienced hematological grade 3 or 4 toxicity, the treatment was delayed one week until the patients recovered from toxicity and then received reduction doses of oxaliplatin and 5-FU in next cycles. Table (2) lists the most frequent treatment related toxicity.

About $16 \%$ of patients experienced grade 2 toxicity of nausea while grade 2 anemia was reported in $27 \%$ of patients. Grade 1 neutropenia was accounted in $65.5 \%$ of patients while grade 3 and 4 neutropenia was accounted in $14.5 \%$, but was complicated by fever or infection in only 2 patients (3.6\%), who needed hospitalization and supportive medical treatment. Although $81.8 \%$ of patients had peripheral neuropathy during treatment Table (3), yet at 18 months $20 \%$ of patients had grade 1 peripheral neuropathy and $7.3 \%$ of patients had grade 2 during the period of follow-up.

The median DFS for whole study group of patients was 7 months (range 4-30 months) Table (4). At 2.5 years, analysis of the entire patients showed that $75 \%$ (95\% CI of DFS: 0.52 to 0.98 ) of patients were alive and disease free, Figure (1). Eighty seven percent of patients with stage II disease are DFS versus $75 \%$ of patients with stage III at end of follow-up ( $\mathrm{p}<0.013$ ), (Figure 2). Nine patients (16\%) had disease relapse or metastasis. Sigmoid colon was the primary tumor site in 7 patients (p 0.001).The median age of relapsed patients was 40 years versus 33 years in non relapsed patients $(\mathrm{P}<0.004)$ and 2 patients presented with signet ring pathological subtype $(\mathrm{P}<0.02)$, the other patients variable showed no significant difference between the two groups.

By multi-variate analysis, shorter DFS was detected among male patients $(\mathrm{P}<0.013)$, Pathological signet ring appearance of the tumor $(\mathrm{P}<0.027)$ and sigmoid site versus rest of colon $(\mathrm{p}<0.033)$.

Among the 55 patients 50 patients (85\%, 95\% $\mathrm{CI}=0.61$ to 0.99 ) were alive at the last follow up. The median OAS for whole study group of patients was10 months (range 4- 30 months), (Figure 3).

The median OAS of relapsed patients was 9.5 months versus 18 months in non relapsed patients $(\mathrm{P}<0.004)$.

By uni-variate in this cohort of patients, there was no correlation between patient's characteristics and survival except lymph node status. Patients with stage II had higher survival percents than patients with stage III, (90\% vs. $80 \%$, respectively $\mathrm{P}<0.002)$, (Figure 4).

By multi-variate analysis only age of patients was independent variable. Patients $>40$ years had prolonged OAS $(\mathrm{P}<0.015)$. 
Table 1: Patients characteristics.

\begin{tabular}{|c|c|c|}
\hline Characteristics & No. & $\%$ \\
\hline No. of patients & 55 & $100 \%$ \\
\hline Sex: Male & 27 & $49.1 \%$ \\
\hline Female & 28 & $50.9 \%$ \\
\hline Age: Median,( years.) & 35 & \\
\hline$<40$ & 31 & $56.4 \%$ \\
\hline $40-60$ & 21 & $38.1 \%$ \\
\hline$>60$ & 3 & $5.5 \%$ \\
\hline \multicolumn{3}{|c|}{ Performance status (WHO) } \\
\hline 0 & 44 & $80 \%$ \\
\hline 1 & 9 & $16.4 \%$ \\
\hline 2 & 2 & $3.6 \%$ \\
\hline \multicolumn{3}{|l|}{ Extent of invasion } \\
\hline T3 & 31 & $56.4 \%$ \\
\hline $\mathrm{T} 4$ & 24 & $43.6 \%$ \\
\hline \multicolumn{3}{|l|}{ No. of nodes examined } \\
\hline Mean & 8.67 & \\
\hline Median & 12 & \\
\hline Range & $0-30$ & \\
\hline \multicolumn{3}{|l|}{ Nodal status } \\
\hline No & 25 & $45.5 \%$ \\
\hline N1-3 & 14 & $25.5 \%$ \\
\hline $\mathrm{N}>3$ & 16 & $29.0 \%$ \\
\hline \multicolumn{3}{|l|}{ Site of tumor } \\
\hline Rt. colon & 30 & $54.6 \%$ \\
\hline Lt .colon & 12 & $21.8 \%$ \\
\hline Sigmoid & 13 & $23.6 \%$ \\
\hline \multicolumn{3}{|l|}{ Histological grade } \\
\hline 1 & 1 & $1.8 \%$ \\
\hline 2 & 40 & $72.7 \%$ \\
\hline 3 & 14 & $25.5 \%$ \\
\hline Bowel obstruction & 15 & $27.2 \%$ \\
\hline Bowel perforation & 4 & $7.2 \%$ \\
\hline \multicolumn{3}{|l|}{ Stage of disease } \\
\hline II & 25 & $45.5 \%$ \\
\hline III & 30 & $54.5 \%$ \\
\hline
\end{tabular}

Table 2: Adverse events among the study group of patients*.

\begin{tabular}{|c|c|c|c|c|c|c|c|c|}
\hline \multirow{2}{*}{ Adverse event } & \multicolumn{2}{|c|}{ Grade 1} & \multicolumn{2}{|c|}{ Grade 2} & \multicolumn{2}{|c|}{ Grade 3} & \multicolumn{2}{|c|}{ Grade 4} \\
\hline & No. & $\%$ & No. & $\%$ & No. & $\%$ & No. & $\%$ \\
\hline Neutropenia & 36 & 68.4 & 11 & 20 & 6 & 10.9 & 2 & 3.6 \\
\hline Anaemia & 30 & 54.5 & 15 & 27.2 & 2 & 3.6 & - & - \\
\hline Thrombo-cytopenia & 40 & 72.7 & 3 & 5.4 & 1 & 1.8 & - & - \\
\hline Nausea & 46 & 83.6 & 9 & 16.3 & - & - & - & - \\
\hline Diarrhea & 28 & 50.9 & 10 & 18.1 & 3 & 5.4 & - & - \\
\hline Stomatitis & 18 & 32.7 & 6 & 10.9 & - & - & - & - \\
\hline Vomiting & 40 & 72.7 & 6 & 10.9 & - & - & - & - \\
\hline Alopecia & 22 & 40.2 & 2 & 3.6 & - & - & - & - \\
\hline Sensory Neuropathy & 29 & 52.7 & 16 & 29 & - & - & - & - \\
\hline
\end{tabular}


Table 3: Incidence of Neurosensory Symptoms during treatment and follow-up in 55 patients treated with FOLFOX-4.

\begin{tabular}{|c|c|c|c|c|c|c|c|c|}
\hline \multirow{2}{*}{ Grade } & \multicolumn{2}{|c|}{ Month 1 follow-up } & \multicolumn{2}{|c|}{ Month6 follow-up } & \multicolumn{2}{|c|}{ Month12 follow-up } & \multicolumn{2}{|c|}{ Month18 follow-up } \\
\hline & No. & $\%$ & No. & $\%$ & No. & $\%$ & No. & $\%$ \\
\hline 0 & 10 & 18.2 & 29 & 52.7 & 36 & 65.5 & 40 & 72.7 \\
\hline 1 & 29 & 52.7 & 18 & 32.6 & 13 & 23.6 & 11 & 20 \\
\hline 2 & 16 & 29.1 & 8 & 14.5 & 6 & 10.9 & 4 & 7.3 \\
\hline
\end{tabular}

Table 4: Analysis of disease free survival (DFS).

\begin{tabular}{lc}
\hline Follow up period & 18 months \\
\hline Median & 3- 30 months \\
Range & \\
\hline Median DFS & 7 months \\
All patients & 6.5 months \\
Relapsed patients & 8 months \\
Non relapsed patients & $75 \%$ \\
Probability of DFS at 2.5 years & $16 \%$ \\
Relapsed patients 9/55 & \\
\hline Death & $9.1 \%$ \\
With relapse 5/55 & \\
\hline
\end{tabular}

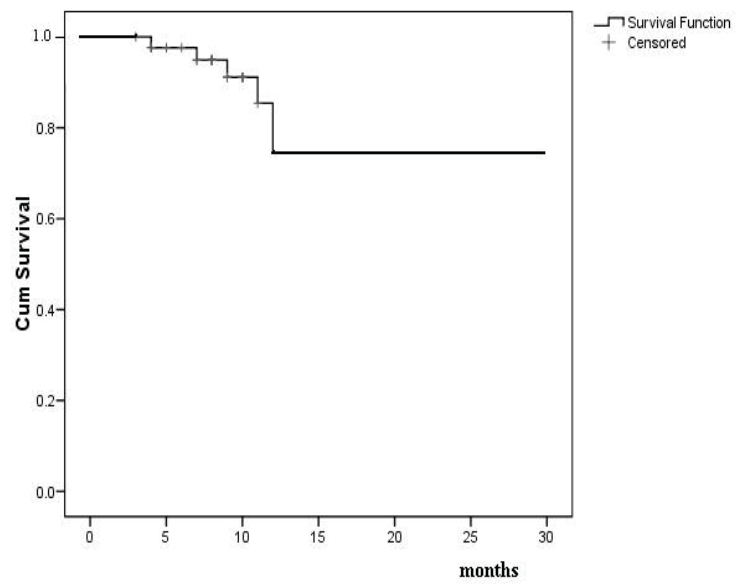

Figure 1: Disease free survival for all cases.

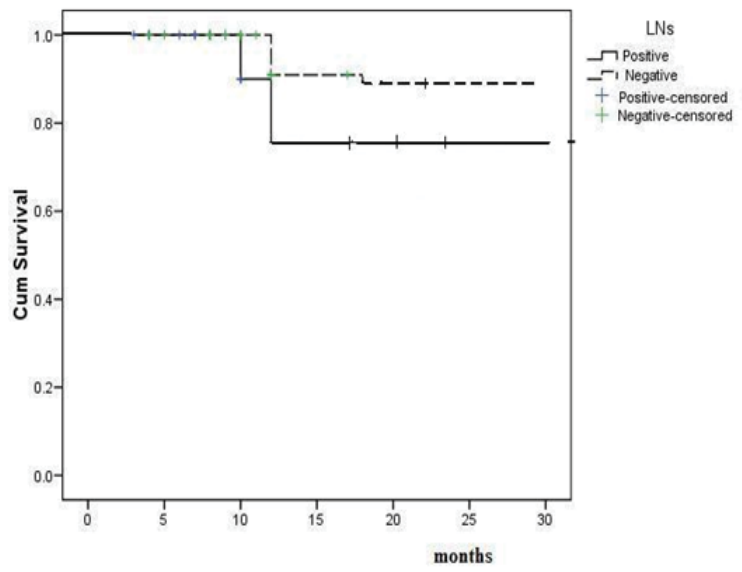

Figure 2: Disease free survival according to lymph nodes status.

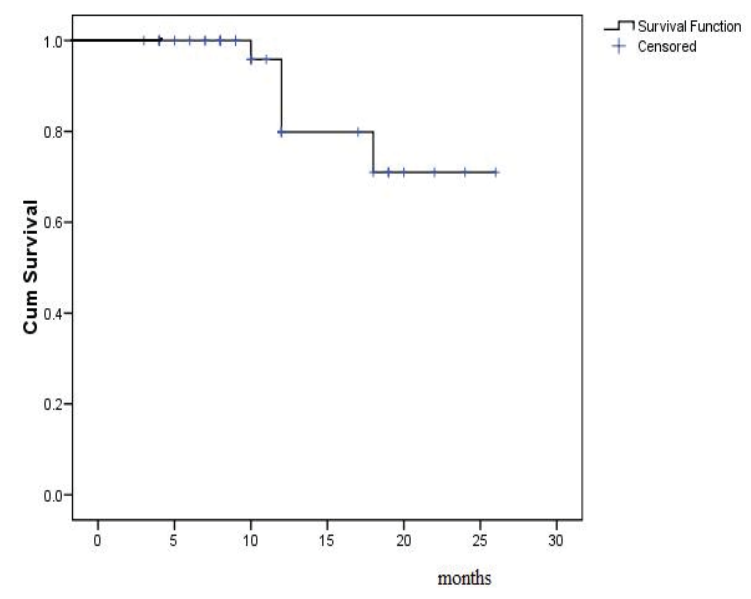

Figure 3: Overall survival for all cases.

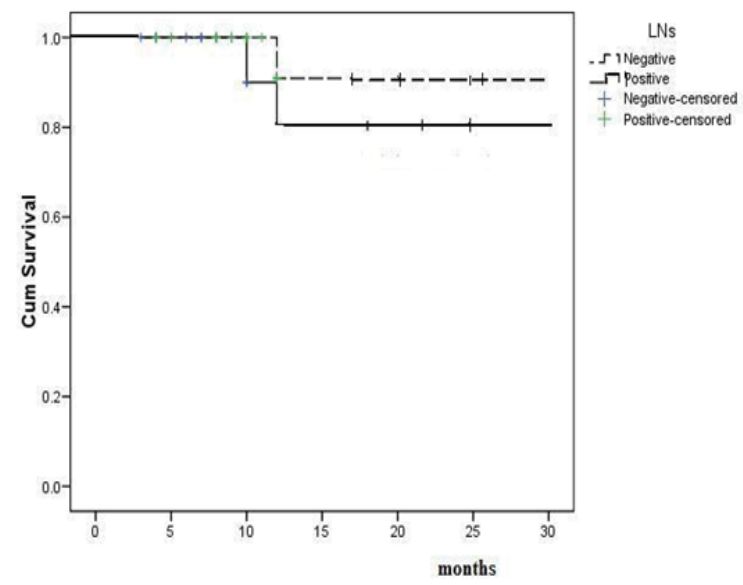

Figure 4: Overall survival according to lymph nodes status.

\section{DISCUSSION}

Studies performed in the late 1980s demonstrated that post operative (5-Fu) plus levamisol improved survival in patients with a resected stage III colon cancer³.

Furtherstudiesperformedinthemid-1990s established (5-Fu) puls (LV) administered for approximately 6 months as standard post operative treatment ${ }^{4-6}$.

De Gramont et al. ${ }^{13}$ reported that addition of oxaliplatin to $\mathrm{Fu}$ and $\mathrm{LV}$ doubled the response rate and prolonged the progression free survival of 
patients with metastatic colorectal cancer $^{13}$. The efficacy and safety of this regimen were confirmed in a large randomized phase III trial, which found that this approach was superior to the combination of irinotecan, Fu and LV (IFL)s, among metastatic colorectal cancer patients ${ }^{14}$.

A clinical trial (MOSAIC) reported by Andre et al. ${ }^{8}$, revealed that adding oxaliplatin to a regimen of bolus and continuous infusion (FU) combined with (LV), (FolFox-4) produced a significant improvement in 3-years DFS compared with the same regimen of $\mathrm{FU} / \mathrm{LV}(78.2 \% \text { VS } 72.9 \%, \mathrm{P}=0.002)^{8}$. At 6 years follow-up confirmed survival was $73 \%$ In FOLFOX -treated patients and $67.4 \%$ in FU/LV treated patients $(\mathrm{P}=0.003)$. However, there is no OS benefit indicated for the stage II colon cancer patients ${ }^{11}$.

The results of NSABP trial (protocol C-07) using oxaliplatin in addition to weekly Roswell Park regime of bolus 5-FU and LV (FLOX) mirrored MOSAIC: 3 year DFS was $76.5 \%$ in FLOX arm and $71.6 \%$ in the bolus FU/LV arm $(\mathrm{p}<0.004)^{10}$.

Our study was tested the efficacy and safety of adjuvant treatment with the regimen of FU/LV plus oxaliplatin in stage III and high risk stage II colon cancer patients in comparison to results of retrospective studies used 5-FU/LV as standard regimen and the primary end point was disease free survival (DFS), since it relates directly to the effect of the treatment under investigation.

Disease free survival (DFS) in our study was 75\% at 2.5 years and falls within the acceptable range reported in most studies of adjuvant treatment of colon cancer investigated FolFox-4 regimen ${ }^{9-11}$. The 3year- DFS reported by MOSAIC and NSABP trials was $78 \%$ and $76.5 \%$, respectively in FOLFOX arm versus $72.9 \%$ and $71.6 \%$ in $\mathrm{FU} / \mathrm{LV}$ arm ${ }^{8,10}$.

Should patient with stage II disease receive adjuvant treatment remains debatable?? Post-surgical therapy for the $25 \%$ of patients diagnosed with CRC remains controversial because $80 \%$ of these patients survive for more than 5 years with surgery alone. However, a subset at high risk for recurrence might benefit from adjuvant therapy ${ }^{17-19}$. In our study we reported $87 \%$ of disease free survival in stage II at the end of follow-up, which is comparable to MOSAIC and NSABP trials, were they accounted $87 \%$ and $86 \%$ as 3 -DFS, respectively In patients with stage II received FOLFOX versus $84 \%$ and $78 \%$ of patients received $\mathrm{FU} / \mathrm{LV}^{8,10}$.
One early study achieved by the Quick and Simple and Reliable (QUASAR) enrolled 3238 patients with stage II CRC who were randomized to observations or adjuvant FU/LV. At 5.5 years, the OS for treated patients was $3.6 \%$ greater than those in the observations arm ( $\mathrm{P}=.001)$; this survival difference was $5.4 \%$ in a subset of patients who were high risk because of large (T4) tumors or vascular invasion 20. Paradoxically, the International Multicenter Pooled Analysis of B2 Colon Cancer Trials (IMPACT B2) failed to demonstrate a statistically significant benefit for stage II colon cancer patients randomized to 5-FU/ LV versus observations16. Most pooled analysis found a positive, but non-significant trend in longer survival in patients with stage II CRC ${ }^{22-23}$.

The National Surgical Adjuvant Breast and Bowel Project (NSABP) concluded that the relative benefits of treatment with FLOX were largely the same for stage II and stage III tumors ${ }^{21}$. In pervious mentioned MOSAIC trial, subset analysis of high -risk patients with stage II CRC found a 7.2\% difference between treatment arms that favored FOLFOX (hazard ratio, $0.76)^{11}$.

Based on this evidence, the American Society of Clinical Oncology (ASCO) recommends that patients with stage II CRC should not routinely receive adjuvant therapy, which may be considered for specific high-risk patients ${ }^{24}$.

The main safety concern regarding the use of oxaliplatin is peripheral neuropathy. Oxaliplatin induces frequent transient distal parathesia during treatment ${ }^{15}$. In MOSAIC trial toxicities were similar in both groups except for grade 3 sensory peripheral neuropathy, which was experienced by $12.4 \%$ of patients who received FOLFOX and $0.2 \%$ of patients who received $\mathrm{FU} / \mathrm{LV}(\mathrm{P}<.001)$. One year after therapy, neuropathy had resolved to grade 0 or 1 in almost all patients, but $1.1 \%$ still had grade 3 neuropathy that decreased somewhat in half of those patients in next 6 months $^{8}$.

FOLFOX -4 regimen was well tolerated among our study group of patients. We observed grade 2 peripheral neuropathy persist to one year in only 6 patients (10.9\%). Among them, two were found to have diabetes that could have caused these symptoms. Although we reported $23.5 \%$ of grade 3 and 4 neutropenia, yet only 2 patients complicated by fever. 
More mature data from the MOSAIC trial published at 2007 and results of confirmatory trial of NASBP C-07 are recommending 5-Fu/LV plus oxaliplatin regimen as a new standard of care as adjuvant therapy after surgery for high risk stage II and III colon cancer patients.10-11 Additionally, the added toxicity and expense of combination therapy, with cost benefit analysis should be consider before widespread use of such therapy in stage II patients.

\section{REFERENCES}

1. Jemal A, Siegel R, Ward E, Murray T, Xu J, Thun MJ. Cancer statistics, 2007. CA Cancer J.Clin. 2007;57(1):43-66.

2. Jemal A, Murray T, Samuels A, Ghafoor A, Ward E, Thun MJ. Cancer statistics, 2003. CA Cancer.J.Clin. 2003 Jan-Feb;53(1):5-26.

3. Moertel CG, Fleming TR, Macdonald JS, Haller DG, Laurie JA, Goodman PJ, et al. Levamisole and fluorouracil for adjuvant therapy of resected colon carcinoma. N.Engl.J.Med. 1990 Feb 8;322(6): 352-8.

4. O’Connell MJ, Mailliard JA, Kahn MJ, Macdonald JS, Haller DG, Mayer RJ, et al. Controlled trial of fluorouracil and low-dose leucovorin given for 6 months as postoperative adjuvant therapy for colon cancer. J.Clin.Oncol. 1997 Jan;15(1):246-50.

5. Wolmark N, Rockette H, Fisher B, Wickerham DL, Redmond C, Fisher ER, et al. The benefit of leucovorin-modulated fluorouracil as postoperative adjuvant therapy for primary colon cancer: Results from National Surgical Adjuvant Breast and Bowel Project protocol C-03. J.Clin.Oncol. 1993 Oct;11(10): 1879-87.

6. Haller DG, Catalano PJ, Macdonald JS, O’Rourke MA, Frontiera MS, Jackson DV, et al. Phase III study of fluorouracil, leucovorin and levamisole in high-risk stage II and III colon cancer: Final report of Intergroup 0089. J.Clin.Oncol. 2005 Dec 1;23(34):8671-8.

7. Lembersky BC, Wieand HS, Petrelli NJ, O’Connell MJ, Colangelo LH, Smith RE, et al. Oral uracil and tegafur plus leucovorin compared with intravenous fluorouracil and leucovorin in stage II and III carcinoma of the colon: Results from National Surgical Adjuvant Breast and Bowel Project Protocol C-06. J.Clin.Oncol. 2006 May 1;24(13):2059-64.

8. Andre T, Boni C, Mounedji Boudiaf L, Navarro M, Tabernero J, Hickish T, et al. Oxaliplatin, fluorouracil and leucovorin as adjuvant treatment for colon cancer. N.Engl.J.Med. 2004 Jun 3;350(23):2343-51.
9. De Gramont A, Boni C, Navarro M, Tabernero J, Hickish T, Topham C, et al. Oxaliplatin/5FU/LV in the adjuvant treatment of stage II and stage III colon cancer: Efficacy results with a median follow-up of 4 years. J.Clin.Oncol. 2005;23:16S (Abstr. 3501).

10. Wolmark N, Wieand S, Lembersky B, Colangelo L, Smith R, Pazdur R. A phase III trial comparing oral UFT to FULV in stage II and III carcinoma of the colon: Results of NSABP Protocol C-06. J.Clin.Oncol. 2004;22:14S (Abstr. 3508).

11. De Gramont A, Boni C, Navarro M, Tabernero J, Hickish T, Topham C, et al. Oxaliplatin/5FU/LV in adjuvant colon cancer: Updated efficacy results of the MOSAIC trial, including survival, with a median follow-up of six years. J.Clin.Oncol. 2007;25:18S (Abstr. 4007).

12. Sanofi-aventis Oncology. Assessing neuropathy: NCI CTC classifications of sensory neuropathy. Available at: http://www.eloxatin.com/hcp/patient_ management/assessing_neuropathy.aspx.

13. De Gramont A, Figer A, Seymour M, Homerin M, Hmissi A, Cassidy J, et al. Leucovorin and fluorouracil with or without oxaliplatin as first-line treatment in advanced colorectal cancer. J.Clin.Oncol. 2000 Aug;18(16):2938-47.

14. Goldberg RM, Sargent DJ, Morton RF, Fuchs CS, Ramanathan RK, Williamson SK, et al. A randomized controlled trial of fluorouracil plus leucovorin, irinotecan and oxaliplatin combinations in patients with previously untreated metastatic colorectal cancer. J.Clin.Oncol. 2004 Jan 1;22(1):23-30.

15. Mamounas E, Wieand S, Wolmark N, Bear HD, Atkins JN, Song K, et al. Comparative efficacy of adjuvant chemotherapy in patients with Dukes' B versus Dukes' C colon cancer: Results from four National Surgical Adjuvant Breast and Bowel Project adjuvant studies (C-01, C-02, C-03 and C-04). J.Clin.Oncol. 1999 May;17(5):1349-55.

16. Efficacy of adjuvant fluorouracil and folinic acid in B2 colon cancer. International Multicentre Pooled Analysis of B2 Colon Cancer Trials (IMPACT B2) Investigators. J.Clin.Oncol. 1999 May;17(5): 1356-63.

17. National Comprehensive Cancer Network (NCCN). NCCN clinical practice guidelines in oncology. Available at: http://www.nccn.org/ professionals/physician_gls/f_guidelines.asp. Accessed Mar 27, 2008.

18. Benson AB III. Selection of patients with stage II disease for adjuvant therapy. Comm.Oncol. 2007;4(Suppl. 3):5-10. 
19. Kopetz S, Freitas D, Calabrich AFC, Hoff PM. Adjuvant chemotherapy for stage II colon cancer. Oncology 2008;22(3):260-70.

20. Quasar Collaborative Group, Gray R, Barnwell J, McConkey C, Hills RK, Williams NS, et al. Adjuvant chemotherapy versus observation in patients with colorectal cancer: A randomised study. Lancet 2007 Dec 15;370(9604):2020-9.

21. Baddi L, Benson A,3rd. Adjuvant therapy in stage II colon cancer: Current approaches. Oncologist 2005 May;10(5):325-31.

22. Gill S, Loprinzi CL, Sargent DJ, Thome SD, Alberts SR, Haller DG, et al. Pooled analysis of fluorouracil- based adjuvant therapy for stage II and III colon cancer: Who benefits and by how much? J.Clin.Oncol. 2004 May 15;22(10):1797-806.

23. Portal vein chemotherapy for colorectal cancer: A meta-analysis of 4000 patients in 10 studies. Liver Infusion Meta-analysis Group. J.Natl.Cancer Inst. 1997 Apr 2;89(7):497-505.

24. Benson AB III, Schrag D, Somerfield MR, Cohen AM, Figueredo AT, Flynn PJ, et al. American Society of Clinical Oncology recommendations on adjuvant chemotherapy for stage II colon cancer. J.Clin.Oncol. 2004;22(16):3408-19. 